Onkologe 2012 $\cdot 18: 298-300$

DOI 10.1007/s00761-012-2223-0

Online publiziert: 10. März 2012

๑) Springer-Verlag 2012

S. Loibl ${ }^{1}$ M. Stuschke ${ }^{2} \cdot$ K. Höffken ${ }^{3}$

${ }^{1}$ German Breast Group Neu-Isenburg und Stadtkrankenhaus Offenbach

${ }^{2}$ Strahlenklinik, Universitätsklinikum Essen

${ }^{3}$ Universitätsklinikum Jena

\title{
Krebs und Schwangerschaft - Fakten und Fiktionen
}

Erkenntnisse und daraus sich ableitende Therapieempfehlungen beruhen auf einer geringen Evidenz. Die höchste Evidenz gibt es für das Mammakarzinom in der Schwangerschaft. Prospektive Kohortenstudien konnten zeigen, dass anthrazyklinhaltige Standardtherapien auch in der Schwangerschaft angewandt werden können. Die ersten internationalen Therapieempfehlungen wurden 2003 erarbeitet. Zeitgleich wurde das Register zur Diagnostik und Therapie des Mammakarzinoms in der Schwangerschaft der German Breast Group (www.germanbreastgoup.de/pregnancy) geschaffen, das die Fälle international registriert und mittlerweile über 450 Schwangere mit Mammakarzinom erfasst.

Etwas später hat die ESGO (European Society für Gynecologic Oncology) ein Register geschaffen, in dem allgemein Karzinomerkrankungen während der Schwangerschaft erfasst werden (www.cancerpregnancy.org). Beide Gruppen arbeiten auf dem Gebiet des Mammakarzinoms eng zusammen. Solche Initiativen helfen, das Wissen zu mehren und den Patientinnen und Therapeuten mehr Sicherheit zu geben und bildeten die Basis für weitere Aktivitäten auf diesem Gebiet.

Im Gegensatz zum Mammakarzinom, das auch während einer Schwangerschaft standardisiert behandeln werden kann, sind die hämatologische Neoplasien, die ein knappes Fünftel der Kar- zinomerkrankungen in der Schwangerschaft ausmachen, eine Herausforderung für den behandelnden Arzt. Er muss in der Regel abwägen, ob es die Erkrankung zulässt, bis zur Lebensfähigkeit des Kindes (noch besser bis mindesten zur 30. SSW) zu warten, um dann mit der für die Mutter optimalen Therapie zu beginnen. Ist dies nicht möglich, müssen sich die Familie und der Arzt gegen das Kind und für die Mutter entscheiden. Dieser fetomaternale Konflikt ist in den meisten Fällen schwer lösbar und stellt ein ethisches Dilemma dar.

\section{\) Am häufigsten sind Mamma- oder Zervixkarzinome sowie hämatologische Erkrankungen}

Eine dritte Kategorie sind die gynäkologischen Karzinome, deren Therapie in den meisten Fällen mit einem Verlust der weiblichen inneren Geschlechtsorgane einhergeht. Eine Schwangerschaft nach einer Krebserkrankung und -therapie, wie sie in den zuerst genannten Fällen noch möglich ist, ist in diesen Fällen ausgeschlossen. Mit dem Rückgang der Inzidenz des Zervixkarzinoms auf weit unter 10.000 Neuerkrankungen pro Jahr sind auch die Fälle in der Schwangerschaft seltener geworden. Hinzukommt, dass der PAP-Abstrich der Zervix in den 
Hier steht eine Anzeige.

黛 Springer 
Mutterschaftsrichtlinien vorgesehen ist und demzufolge die meisten Veränderungen an der Zervix zeitnah mit dem Nachweis der Schwangerschaft vergesellschaftet sind.

Grundsätzlich gilt für jede Behandlung in der Schwangerschaft: so viel wie nötig und so wenig wie möglich, um Mutter und Kind gerecht zu werden.

\section{》) Eine Schwangerschaft nach Malignomerkrankungen muss individuell beurteilt werden}

Die Frage ob eine Schwangerschaft nach erfolgreich behandelter Krebserkrankung möglich ist, beschäftigt viele Paare und muss immer individuell beurteilt werden. Die Antwort ist in erster Linie abhängig von der Erkrankung und der damit verbundenen Therapie. Wie hoch ist das Rückfallrisiko? Hat die Therapie die Fruchtbarkeit reduziert? Steigt durch die Schwangerschaft das Rezidivrisiko? Hat das Kind auch nach der Therapie mit Schädigungen durch die Therapie zu rechnen? Diese und mehr Fragen kommen auf den behandelnden Arzt zu und häufig werden die Paare mit der Information nach Hause geschickt, dass eine Schwangerschaft nicht anzustreben ist. Jedoch gibt es mittlerweile gute Daten, die zeigen, dass in den meisten Fällen von einer Schwangerschaft nicht abgeraten, evtl. sogar zugeraten werden sollte.

Das vorliegende Schwerpunktheft versucht, aus Expertensicht Antworten anhand existierender Fakten auf Fragen zu geben. Der Leser mag erkennen, dass eine Vielzahl von Fragen, die ihm seine Patientinnen im praktischen Alltag stellen, nicht auf der Basis eines gesicherten Kenntnistands beantwortet werden können, insbesondere dort nicht, wo persönliche Lebenspläne, Maßstäbe und Einstellungen Eingang in die Meinungsbildung und Entscheidung finden. Das heißt aber nicht, dass Fakten durch Fiktionen ersetzt werden dürfen.

Krebs und Schwangerschaft - Schwangerschaft und Krebs, ein ungleiches Paar, das aber in Zukunft mehr Beachtung erhalten muss.

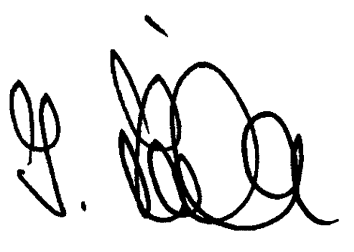

Sibylle Loibl

Für die Herausgeber des Schwerpunkthefts

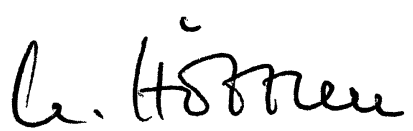

Klaus Höffken

Für die Herausgeber

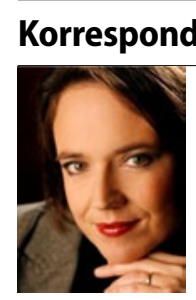

\section{DKG-Internetportal: Nachlese DKK 2012}

Ende Februar fand in Berlin unter dem Motto "Qualität sichern - Forschung fo(e)rdern" der 30. Deutsche Krebskongress (DKK) statt. Über 9.000 Teilnehmer kamen zum größten und wichtigsten onkologischen Fachkongress im deutsprachigen Raum, um intensiv über die aktuellen Entwicklungen in der Onkologie zu diskutieren.

Journalistisch begleitet wurde der DKK vom Redaktionsteam des Internetportals der Deutschen Krebsgesellschaft (DKG). Eine Vielzahl von Berichten, Videos und Expertengesprächen geben Einblicke in die relevanten Ergebnisse des Kongressgeschehens.

Im Fokus stehen hierbei unter anderem Aspekte der Gesundheitspolitik, wie beispielsweise in der Berichterstattung über die DKK-Schwerpunktsitzungen zu den Themen Forschungspolitik, Qualitätssicherung, Leben mit Krebs und Nationaler Krebsplan oder bei der Aufzeichnung des neu etablierten „Parlamentarischen Abends". Andererseits fokussiert das Internetportal auf interessante Entwicklungen im Bereich von Medizin und Forschung und lässt nationale und internationale Experten wie die Sprecher der DKK-Keynote-Lectures und die Sprecher der DKG-Arbeitsgemeinschaften umfassend zu Wort kommen.

Alle Interviews, Berichte und Videoaufzeichnungen sind ab sofort online abrufbar unter www.krebsgesellschaft.de/dkk_2012.

Quelle: Deutsche Krebsgesellschaft e.V., www.krebsgesellschaft.de 